\title{
Biomarkers of early chronic obstructive pulmonary disease (COPD) in smokers and former smokers. Protocol of a longitudinal study
}

\author{
Mikael Truedsson ${ }^{1}$, Johan Malm²,3, K. Barbara Sahlin²,3* May Bugge ${ }^{1}$, Elisabet Wieslander ${ }^{3}$, Magnus Dahlbäck $^{3,4}$,
} Roger Appelqvist ${ }^{3,4}$, Thomas E. Fehniger ${ }^{3,4 \wedge}$ and György Marko-Varga ${ }^{3,4,5}$

\begin{abstract}
Background: Chronic obstructive pulmonary disease (COPD) is an irreversible disease, diagnosed predominantly in smokers. COPD is currently the third leading cause of death worldwide. Far more than $15 \%$ of smokers get COPD: in fact, most develop some amount of pulmonary impairment. Smoking-related COPD is associated with both acute exacerbations and is closely correlated to comorbidities, such as cardiovascular disease and lung cancer. The objective of our study (KOL-Örestad) is to identify biomarkers in smokers and ex-smokers, with early signs of COPD, and compare these biomarkers with those of non-smokers and healthy smokers/ex-smokers. The participants in the study are recruited from Örestadskliniken, a primary health care clinic in Malmö, Sweden.

Methods: Two hundred smokers and ex-smokers diagnosed with COPD with airflow restriction according to GOLD stages 1-4 will be included and compared with 50 healthy never-smokers, and 50 healthy smokers/ex-smokers without airflow restriction (total $n=300$ ). The age distribution is $35-80$ years. The participants undergo a health examination including medical history, smoking history, lung function measurements, and respond to a "Quality of Life" questionnaire. Blood samples are drawn every 6 months during a period of 5 years. Additional blood sample collection is performed if participants are experiencing an exacerbation. The blood fractions will be analyzed by standard clinical chemistry assays and by proteomics utilizing mass spectrometry platforms. Optimal sample integrity is ensured by rapid handling with robotic biobank processing followed by storage at $-80^{\circ} \mathrm{C}$. The study has been approved by the Regional Ethical Review Board in Lund (http://epn.se/en), (Approval number: DNR 2013/480), and registered at the $\mathrm{NIH}$ clinical trial registry (http://clinicaltrials.gov).

Results and discussion: Currently, 220 subjects are enrolled in the study.

Conclusions and future directions: The study design will enable discovery of new biomarkers by using novel mass spectrometric techniques that define early changes of COPD. Such panels of novel biomarkers may be able to distinguish COPD from closely related diseases, co-morbidities, and contribute to an increased understanding of these diseases.
\end{abstract}

Keywords: COPD, Clinical study, Biomarkers, Proteomics, Biobanking

\footnotetext{
*Correspondence: barbara.sahlin@med.lu.se

$\wedge$ Deceased

${ }^{3}$ Centre of Excellence in Biological and Medical Mass Spectrometry,

Biomedical Centre D13, Lund University, 22184 Lund, Sweden

Full list of author information is available at the end of the article
}

\section{垈 Springer}

(C) 2016 Truedsson et al. This article is distributed under the terms of the Creative Commons Attribution 4.0 International License (http://creativecommons.org/licenses/by/4.0/), which permits unrestricted use, distribution, and reproduction in any medium, provided you give appropriate credit to the original author(s) and the source, provide a link to the Creative Commons license, and indicate if changes were made. 


\section{Background}

Chronic obstructive pulmonary disease (COPD) is an irreversible disease, diagnosed predominantly in smokers. COPD is currently the third leading cause of death worldwide [1, 2]. It is a heterogeneous inflammatory disease characterized by different phenotypes, such as airflow limitation that is not fully reversible, chronic sputum production (chronic bronchitis) and destruction of parenchymal lung tissue (emphysema) [2]. Active smoking is the main risk factor for developing COPD. Far more than $15 \%$ of smokers get COPD: in fact, most develop some amount of pulmonary impairment [3]. Smoking-related COPD is associated with acute exacerbations and is closely correlated to comorbidities, such as cardiovascular disease and lung cancer [2, 4]. The number of inflammatory cells and mediators is increased in the lungs and can be detected in bronchoalveolar lavage, biopsies and blood [5]. The pathophysiology of these systemic manifestations is unclear; however, several attempts to study biomarkers closely associated with COPD phenotypes have been performed, both in crosssectional and longitudinal studies. Standardized methodology and strict quality control, replication of data in different cohorts, as well as robust and cost-effective clinical tests are needed [6]. Significant differences in biomarkers when comparing patients with COPD with former or non-smoking controls have been described [7]. Some biomarkers showed large variability within the three months study period. Plasma fibrinogen was the most 'reliable' analyte and was elevated, along with plasma CRP, in COPD patients experiencing exacerbations compared to COPD patients without exacerbations.

However, expiratory volume in one second $\left(\mathrm{FEV}_{1}\right)$ is the most widely used parameter to evaluate the disease severity with increasing airflow limitations $[2,8]$. The ratio of $\mathrm{FEV}_{1}$ to forced vital capacity (FVC) is measured with spirometry and is currently most frequently used to classify the disease state of COPD according to the Global initiative for Obstructive Lung Disease (GOLD) stages 1-4 [9]. Yet, $\mathrm{FEV}_{1}$ correlates poorly to clinical symptoms in early stages of COPD, but slightly better in later stages of the disease [2]. A study from Evaluation of COPD Longitudinally to Identify Predictive Surrogate Endpoints (ECLIPSE) found that the degree of airflow limitation does not reflect the heterogeneity of COPD. Additionally, a substantial part of the COPD patients, even with severe airflow obstruction, did not complain of any symptoms [10]. Furthermore, there are recent suggestions to classify structural changes in COPD using CT-imaging of morphological changes in pulmonary tissue tracts [11]. The most recent GOLD classification includes both symptoms and "Quality of Life" (QoL) in addition to lung function [12].
COPDGene is another front line study that was first to define both genetic factors implicated in COPD, as well as to investigate structural pulmonary changes with CT. The original cross-sectional study, which started 2008, has been extended with several follow-up studies to detect changes during disease progression [13].

Over the last decade, several studies have attempted to identify new biomarkers of COPD in blood, biomarkers both for diagnosing the disease as well as to predict exacerbations and to classify disease states [14, 15]. A number of studies on COPD plasma samples analyzed by mass spectrometry reported candidate protein biomarkers [16-18]. However, the current COPD studies available on patient blood samples analyzed by mass spectrometry are limited and there is still a lack of reliable biomarkers, a fact that complicates personalizing the care of patients with COPD.

The objective of this study (KOL-Örestad) is to identify circulating biomarkers in smokers and former smokers with early signs of COPD and compare these biomarkers with those of non-smokers and healthy smokers/ ex-smokers. This could facilitate early diagnosis, evaluation of treatment outcome and more accurately classify patients with COPD. Participants are recruited from Örestadskliniken, a primary health care clinic in Malmö, Sweden. KOL-Örestad is the first COPD study to combine a primary health care, with a centralized large scale state hospital in a collaborative effort with academia.

\section{Methods}

\section{Study outline}

This study is a close collaboration between a primary health care clinic (Örestadskliniken) in Malmö, the Skåne University Hospital in Malmö and Lund, and the Centre of Excellence in Biological and Medical Mass Spectrometry (CEBMMS, http://cebmms.lu.se) at Lund University, as depicted in Fig. 1, where the infrastructure of the study is illustrated. The study was initiated in 2014 and will continue for five years. The collaboration provides efficient blood sample access and high quality samples. The study has been approved by the Regional Ethical Review Board in Lund (http://epn.se/en), (Approval number: DNR 2013/480), and registered at the NIH clinical trial registry (http://clinicaltrials.gov).

Blood biomarkers from two hundred smokers and exsmokers with diagnosed COPD according to GOLD stage 1-4 will be compared to those of a control group of 50 healthy never-smokers and 50 healthy smokers/ex-smokers (Table 1). Exclusion criteria include presence of a chronic inflammatory disease or treatment with steroids or any other immunomodulatory treatment that is unrelated to exacerbations of COPD. These conditions may otherwise interfere with the biomarkers measured in the study. 


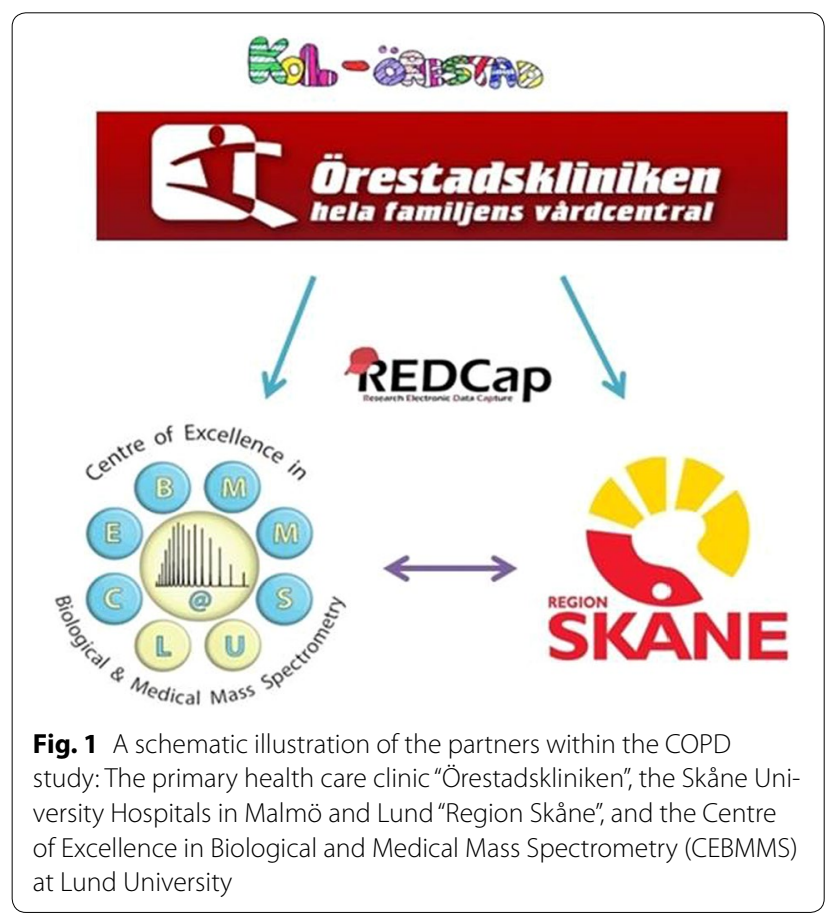

The participants will undergo health examinations including heart and lung, as well as blood pressure and spirometry. They will also fill out a QoL questionnaire regarding smoking habits, COPD symptoms, disease history and current medication. Blood samples are collected every six months, which are processed and stored as individual samples of plasma, buffy coat, red blood cells, serum and whole blood. Blood samples are collected in separate tubes (vacutainers) containing sodium heparin, sodium citrate or EDTA (ethylenediaminetetraacetic acid) as anticoagulants, or clot activator for serum separation. Separate plasma tubes are used to analyze a defined list of biomarkers at the Clinical Chemistry Laboratory at Skåne University Hospital in Malmö. Additional blood sample collection is performed when participants experience exacerbations.
All data are stored in a clinical database, using Research Electronic Data Capture (REDCap) as a data capture tool [19]. The blood samples will be stored at $-80{ }^{\circ} \mathrm{C}$ in the biobank and subsequently be analyzed by liquid chromatography mass spectrometry (nano LC-MS/MS) techniques at CEBMMS, Lund University. The current status of the study recruitment and the participant distribution according to the GOLD standards are shown in Fig. 2. Currently, there are more participants in the healthy group and in GOLD stage 2, while participants in GOLD stage 4 are being recruited.

\section{Clinical samples and biobanking}

The biobanking workflow has previously been developed at CEBMMS for rigorous standardization for large scale biobanking [20-23]. The blood samples are collected at the primary health care clinic and transported by a courier to the biobank at CEBMMS for aliquoting and storage. Four vacutainers (EDTA, citrate, heparin, serum) are centrifuged at $2000 \mathrm{~g}$ for $10 \mathrm{~min}$ at room temperature in order to separate blood into three fractions: plasma, buffy coat and red blood cells. The automatic aliquoting of samples is performed by a robot (HAMILTON robot MicroLab Starlet, Hamilton, Bonaduz AG, Switzerland) that aliquots $70 \mu \mathrm{L}$ of the sample per vial into half of the sample rack of a 384-well plate. Thus, blood from two subjects can be stored in parallel into one 384-well plate. The 1-D barcode on the vials arriving from the clinic are matched with another 1-D barcode on the 384-well plate. Each individual aliquot is subsequently labeled with a 2-D barcode encrypted for each patient and sample type to provide full traceability of the samples. The aliquot vials are then sealed with aluminum foil and punched individually. All plates are stored at $-80^{\circ} \mathrm{C}$ in a fully automated biobank storage unit (LICONIC freezer STT1k5 ULT, Liconic AG, Mauren, Liechtenstein) that is able to store clinical samples at a density of five million tubes [23]. This procedure, from sample collection to storage in the biobank, takes less than $2 \mathrm{~h}$, which ensures high sample

Table 1 Study participant distribution according to the GOLD standards

\begin{tabular}{|c|c|c|}
\hline $\begin{array}{l}\text { Study groups by pack years } \\
\text { and air flow limitation }\end{array}$ & $\begin{array}{l}\text { Pack years } 1 \text { pack year }=20 \text { cigarettes } \\
\text { a day in } 1 \text { year }\end{array}$ & $\begin{array}{l}\text { Airflow limitation } \\
\text { FEV }_{1} / F V C<70 \%\end{array}$ \\
\hline Healthy never smokers $n=50$ & 0 & No \\
\hline Healthy smokers/ex-smokers $n=25$ & $<20$ & No \\
\hline At risk smokers/ex-smokers $n=25$ & $>20$ & No \\
\hline GOLD stage 1 smokers/ex-smokers $n=75$ & $>20$ & $\mathrm{FEV}_{1} \geq 80 \%$ \\
\hline GOLD stage 2 smokers/ex-smokers $n=75$ & $>20$ & $50 \% \leq \mathrm{FEV}_{1}<80 \%$ \\
\hline GOLD stage 3 smokers/ex-smokers $n=25$ & $>20$ & $30 \% \leq \mathrm{FEV}_{1}<50 \%$ \\
\hline GOLD stage 4 smokers/ex-smokers $n=25$ & $>20$ & $\mathrm{FEV}_{1}<30 \%$ \\
\hline
\end{tabular}

The enrolled subjects will be divided in the following study groups. The total number of participants will be 300 , including 50 healthy never smokers, 25 healthy smokers and ex-smokers, 25 at risk smokers and ex-smokers, 200 participants with GOLD stage 1-4 divided in 75 with GOLD stage 1 and 2 respectively (smokers and ex-smokers) and 25 with GOLD stage 3 and 4 respectively COPD participants (smokers and ex-smokers) 


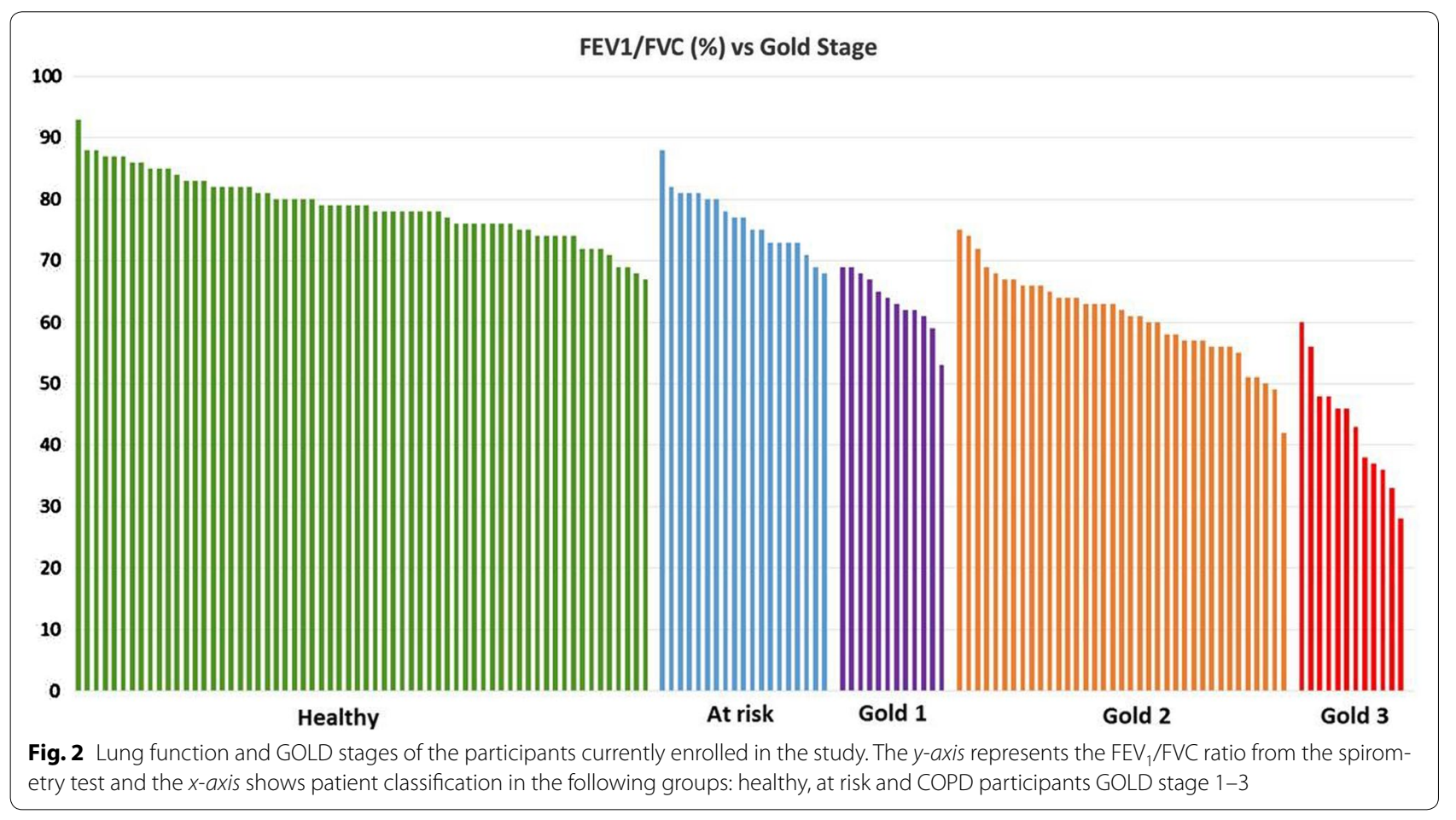

quality and safe handling of the samples. By producing multiple aliquots from the same sample, the freezingand-thawing procedure is avoided, which may otherwise decrease sample quality. Each participant is assigned a unique identifier (Study ID) allowing all samples and data to be handled anonymously. The study ID can only be deciphered by clinicians at Örestadskliniken. For analysis, the aliquot vials can be retrieved via electronic command through the computerized biobanking system.

\section{Clinical database}

The data acquired are stored in a database, KOL-Örestad database, including clinical patient data, blood pressure, spirometry, and a QoL questionnaire detailing smoking habits, COPD symptoms, and disease history. By use of the barcode or patient study ID, all clinical demographics, type of blood samples, and analyzed output data stored in the database, can be traced from each patient. REDCap from Vanderbilt University, TN, USA is selected as an electronic data capture tool for collection and managing the study data. REDCap provides a flexible and easily programmable interface especially adapted for clinical studies [19]. REDCap has user-specified input modules for a specific cluster of variables, e.g. modules on: QoL, blood sampling, spirometry, without restriction in time and numbers. User allowance can be adjusted for different users, e.g. clinicians and researchers. This ensures patient integrity and maximizes flexibility of the platform.
All patient data that are handled outside the health care sector is encrypted and handled anonymously.

REDCap also functions as a planning instrument for clinicians, facilitating the planning of the number of patient follow ups each participant has completed. This gives the involved clinicians and researchers an excellent overview of all participants and their level of completion in the study.

Health Information System (HIS) provides the foundation for decision-making and is comprised of four key functions: data generation, compilation, analysis and synthesis, and communication and use (Fig. 3). Data from the health care sector is collected within HIS as well as analyzed and controlled for quality, relevance, and timeliness [24].

\section{Data acquisition}

The blood samples will be prepared for data acquisition by mass spectrometry by dilution, addition of chicken lysozyme, solubilization, reduction, alkylation, digestion with trypsin, spiking with heavy labeled peptide standards and centrifugation. More details of the preparations will be optimized and validated for a multiple reaction monitoring (MRM) workflow for the potential protein biomarkers from the collected blood samples, which will be analyzed by liquid chromatography mass spectrometry (nano LC-MS/MS). The confirmation of protein identities will be carried out based on a library obtained from 


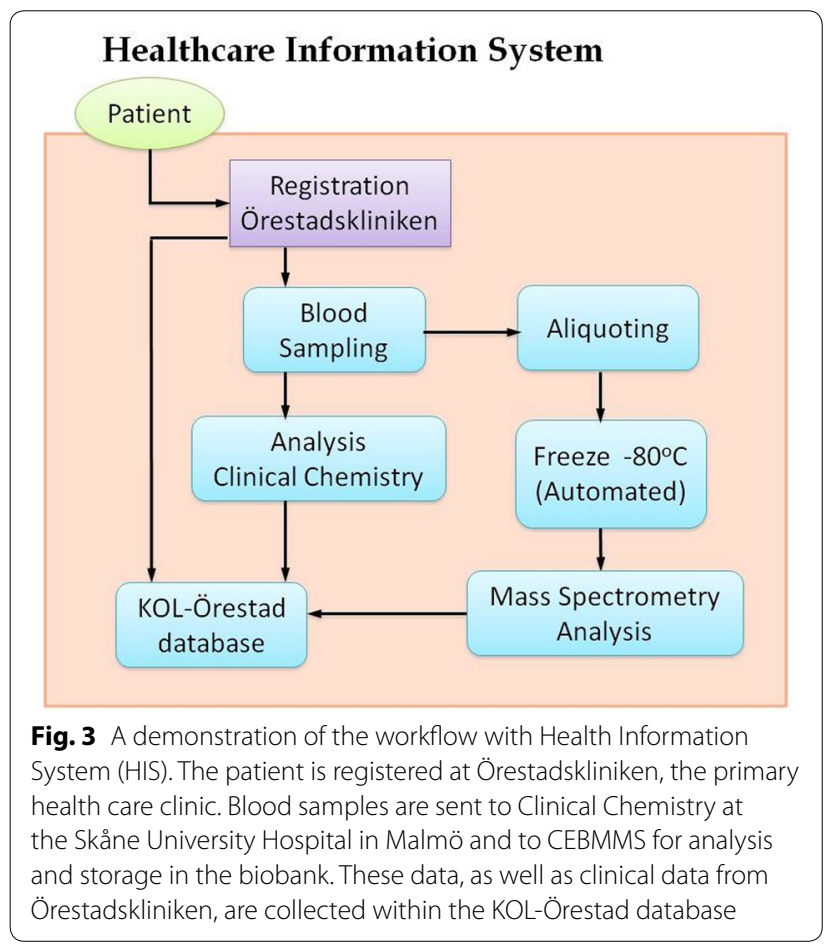

Proteome database search (v 1.4, Thermo Scientific, San José, CA) and prior data dependent analysis (DDA) of pooled plasma samples from COPD patients and healthy participants.

The data input and logistical work flow is depicted in Fig. 3, followed by data output from resulting assay readouts. Separate blood samples will be analyzed by standard clinical chemistry assays.

\section{Results and discussion}

The aim of the study includes novel biomarker technology development on blood collections of patients with COPD and additional sample collections during exacerbations. COPD patients who also have cardiovascular diseases and lung cancer are included in the study, enabling cross over multi-factorial effects and co-morbidities to be investigated. Currently, 220 subjects are enrolled in the study. Because screening with spirometry is not regularly performed in the primary health care clinic on the aging population, new available tests would greatly benefit diagnostics. Biobanks are highly important for the introduction of personalized medicine in future health care $[25,26]$. Preservation of clinical samples collected daily is an essential undertaking, because each sample is unique and has the potential to facilitate future research and diagnostics.

Quantitative mass spectrometry provides an excellent method to identify biomarkers that can be used in personalized medicine. Mass spectrometry is very sensitive, which makes sample quality even more important. The present system generates aliquots of $70 \mu \mathrm{L}$ in a 384-well plate using an automated sample processing platform. This minimizes the probability of methodological errors and improves sample quality by avoiding the use of previously thawed samples [23]. In our workflow, we have developed a method for safe handling of samples to maximize sample preservation, which is robust and well suited for clinical studies.

\section{Conclusion and future directions}

In this study, the disease progression will be followed longitudinally for each patient. Novel disease biomarkers identified by proteomics (significantly up- or down-regulated due to disease state) will be related to measured clinical parameters. The study design will enable the discovery of new biomarkers that define early changes of COPD, distinct from markers of closely related diseases, such as cardiovascular disease and lung cancer. Such panels of novel biomarkers can contribute to an increased understanding of these diseases.

\section{Abbreviations}

GOLD: global initiative for chronic obstructive lung disease; COPD: chronic obstructive pulmonary disease; QoL: quality of life; HIS: health information system; nano LC-MS/MS: liquid chromatography mass spectrometry.

\section{Authors' contributions}

MT, MB: patient recruitment and physical examination, MB, MT, JM: clinical input, JM, TEF, EW, GMV, MD: study outline, KBS, GMV, MD, RA, EW, JM: manuscript preparation, MD, RA: study logistics and programming, GMV, MD, RA, EW: data analysis, All authors read and approved the final manuscript.

\section{Author details}

${ }^{1}$ Örestadskliniken, Eddagatan 4, 21767 Malmö, Sweden. ${ }^{2}$ Section for Clinical Chemistry, Department of Translational Medicine, Lund University, Skåne University Hospital Malmö, 20502 Malmö, Sweden. ${ }^{3}$ Centre of Excellence in Biological and Medical Mass Spectrometry, Biomedical Centre D13, Lund University, 22184 Lund, Sweden. ${ }^{4}$ Clinical Protein Science and Imaging, Biomedical Centre, Department of Biomedical Engineering, BMC D13, Lund University, 22184 Lund, Sweden. ${ }^{5}$ First Department of Surgery, Tokyo Medical University, 6-7-1 Nishishinjiku, Shinjiku-ku, Tokyo 160-0023, Japan.

\section{Acknowledgements}

The authors gratefully acknowledge support from Medical Training Research Agreement from Region Skåne and Alfred Österlunds Stiftelse.

\section{Competing interests}

The authors declare that they have no competing interests.

Received: 9 December 2015 Accepted: 16 February 2016 Published online: 07 March 2016

\section{References}

1. World Health Organization. Fact Sheet http://www.who.int/mediacentre/ factsheets/fs310/en/.Accesssed 25 Nov2015

2. Rennard SI, Drummond MB (2015) Early chronic obstructive pulmonary disease: definition, assessment, and prevention. Lancet 385(9979):1778-1788

3. Rennard SI, Vestbo J (2006) COPD: the dangerous underestimate of 15\%, Lancet 367(9518):1216-1219 
4. Decramer M, Janssens W (2013) Chronic obstructive pulmonary disease and comorbidities. Lancet Respir Med. 1(1):73-83

5. Thomsen M, Dahl M, Lange P, Vestbo J, Nordestgaard BG (2012) Inflammatory biomarkers and comorbidities in chronic obstructive pulmonary disease. Am J Respir Crit Care Med 186(10):982-988

6. Faner R, Tal-Singer R, Riley JH, Celli B, Vestbo J, MacNee W et al (2014) ECLIPSE Study Investigators. Lessons from ECLIPSE: a review of COPD biomarkers. Thorax 69(7):666-672

7. Dickens JA, Miller BE, Edwards LD, Silverman EK, Lomas DA, Tal-Singer R (2011) Evaluation of COPD longitudinally to identify surrogate endpoints (ECLIPSE) study investigators. COPD association and repeatability of blood biomarkers in the ECLIPSE cohort. Respir Res 12:146

8. Loi ALT, Hoonhorst SJ, Franciosi L, Bischoff R, Hoffmann RF, Heijink I et al (2013) Acute and chronic inflammatory responses induced by smoking in individuals susceptible and non-susceptible to development of COPD: from specific disease phenotyping towards novel therapy. Protocol of a cross-sectional study. BMJ Open. 3(2):002178

9. Global Initiative for Chronic Obstructive Lung Disease. Spirometry for Health Care Providers - Global Initiative for Chronic Obstructive Lung Disease (GOLD). Global Initiative for Chronic Obstructive Lung Disease (GOLD). 2010. http://www.goldcopd.org/uploads/users/files/GOLD_ Spirometry_2010.pdf Accessed 25 Nov 2015

10. Agusti A, Calverley PM, Celli B, Coxson HO, Edwards LD, Lomas DA et al (2010) Evaluation of COPD longitudinally to identify predictive surrogate endpoints (ECLIPSE) investigators. Characterisation of COPD heterogeneity in the ECLIPSE cohort. Respir Res 11:122

11. Lynch DA, Austin JH, Hogg JC, Grenier PA, Kauczor HU, Bankier AA et al (2015) CT-definable subtypes of chronic obstructive pulmonary disease: a statement of the Fleischner society. Radiology 277(1):192-205

12. Global Initiative for Chronic Obstructive Lung Disease. Pocket Guide to COPD Diagnosis, Management, and Prevention-a guide for health care professionals. Global Initiative for Chronic Obstructive Lung Disease. Updated 2015. http://www.goldcopd.org/uploads/users/files/GOLD_ Pocket_2015_Feb18.pdf Accessed 25 Nov 2015

13. Regan EA, Hokanson JE, Murphy JR, Make B, Lynch DA, Beaty TH (2010) COPD Gene investigators. Genetic epidemiology of COPD (COPD Gene) study design. COPD. 7(1):32-43

14. Han MK, Kazerooni EA, Lynch DA, Liu LX, Murray S, Curtis JL (2011) COPD Gene Investigators. Chronic obstructive pulmonary disease exacerbations in the COPD gene study: associated radiologic phenotypes. Radiology 261(1):274-282
15. Shaw JG, Vaughan A, Dent AG, O'Hare PE, Goh F, Bowman RV et al (2014) Biomarkers of progression of chronic obstructive pulmonary disease (COPD). J Thorac Dis. 6(11):1532-1547

16. Bortner JD Jr, Richie JP Jr, Das A, Liao J, Umstead TM, Stanley A et al (2011) Proteomic profiling of human plasma by iTRAQ reveals downregulation of ITI HC3 and VDBP by cigarette smoking. J Proteome Res 10(3):1151-1159

17. Merali S, Barrero CA, Bowler RP, Chen DE, Criner G, Braverman A (2014) Analysis of the plasma proteome in COPD: novel low abundance proteins reflect the severity of lung remodeling. COPD. 11(2):177-189

18. Bandow JE, Baker JD, Berth M, Painter C, Sepulveda OJ, Clark KA et al (2008) Improved image analysis workflow for 2-D gels enables large-scale 2-D gel-based proteomics studies-COPD biomarker discovery study. Proteomics 8(15):3030-3041

19. Harris PA, Taylor R, Thielke R, Payne J, Gonzalez N, Conde JC (2009) Research electronic data capture (REDCap) — A metadata-driven methodology and workflow process for providing translational research informatics support. J Biomed Inform 42(2):377-381

20. Malm J, Fehniger TE, Danmyr P, Végvári A, Welinder C, Lindberg H et al (2013) Biobanking work flow standardization—developments providing sample integrity. J Proteomics. 95:38-45

21. Malm J, Danmyr P, Nilsson R, Appelqvist R, Végvári A, Marko-Varga G (2013) Blood sample standardization developments for large scale biobanking. J Proteome Res 12:3087-3092

22. Fehniger TE, Boja ES, Rodriguez H, Baker M, Marko-Varga G (2014) Four areas of engagement requiring strengthening in modern proteomics today. J Proteome Res 13(12):5310-5318

23. Malm J, Linberg $H$, Erlinge $D$, Appelqvist $R$, Yokaleva $M$, Welinder C et al (2015) Semi-automated biobank sample processing with 384 high density sample tube robot used in cancer and cardiovascular studies. Clin Transl Med. 4(1):67

24. World Health Organization. Health Metrics Network Framework and Standards for Country Health Information Systems. 2008. http://www. who.int/healthinfo/statistics/toolkit_hss/EN_PDF_Toolkit_HSS_InformationSystems.pdf Accessed 25 Nov 2015

25. Riegman PHJ, Morente MM, Betsou F, De Blasio P, Geary P (2008) Marble arch int working $G$ biobanking for better healthcare. Mol Oncol. 2(3):213-222

26. Khleif SN, Doroshow JH, Hait WN (2010) AACR-FDA-NCI Cancer Biomarkers Collaborative Consensus Report: advancing the use of biomarkers in cancer drug development. Clin Cancer Res 16:3299-3318

\section{Submit your manuscript to a SpringerOpen ${ }^{\circ}$ journal and benefit from:}

- Convenient online submission

- Rigorous peer review

- Immediate publication on acceptance

- Open access: articles freely available online

- High visibility within the field

- Retaining the copyright to your article

Submit your next manuscript at $>$ springeropen.com 